\title{
ANALYSIS OF COGNITIVE PROCESSES OF A DESIGNER AS THE FOUNDATION FOR SUPPORT TOOLS
}

\author{
MASAKI SUWA, JOHN GERO, TERRY PURCELL \\ Key Centre of Design Computing, \\ Department of Architectural and Design Science \\ The University of Sydney, Australia, NSW 2006
}

\begin{abstract}
Drawing sketches while designing plays an essential role in crystallising design ideas. Past literature has revealed important aspects of sketching that facilitate design processes. There has been no empirical study, however, to reveal how sketches are involved in the very process in which the structures of designers' actions are dynamically formed. We have devised a general scheme to code designers' actions belonging to different cognitive levels. Based on the scheme, we analyzed the process of a practicing architect. We found evidence that suggests the following insights. First, the role of sketching is mostly to leave ideas on paper for later inspection and thus perception, rather than to give simultaneous incentives for perception. Second, the role of perception is to stimulate functional thoughts, especially in the phase of detailed exploration. Third, therefore, design behaviors are situated in the physical setting in which designers draw and perceive. Based on these insights, we have proposed the concept of a set of different types of design support tools. They should assist designers' actions to draw and perceive while maintaining the situatedness of design behaviors.
\end{abstract}

\section{Introduction}

Design is a kind of apprenticeship in which skills and expertise are acquired after learning basic techniques, assimilating domain knowledge, and inspecting past good examples. Acquired expertise, however, is often tacit and implicit. Even a skilled designer cannot articulate what kind of expertise he/she uses in designing and how. This is one of the major problems in design, especially in the pedagogical sense. We lack the design methodology to improve the quality of processes and products as well as to implement computational support tools.

Among examples of the tacitness of design expertise is the use of sketches. Designers usually begin with freehand sketches to come up with promising ideas, and later turn to using drafting/CAD tools and to building mockups, for the purposes of visualization, comparison and implementation of the ideas. Why 
are freehand sketches essential for crystallizing ideas in a preliminary design stage? How do skilled designers capitalize on the use of sketches in their processes?

Schon and Wiggins (1992) pointed out unexpected discoveries as a benefit of using sketches. Externalizing a set of ideas on paper forces spatial organization and specificity in Stenning and Oberlander's sense (1995), which, in turn, by inspection, may lead to new discoveries in an unintended way. Another benefit is the effect of re-interpretation. Revisiting their own depictions sketched, designers do not necessarily interpret them with the same meanings as the original, but sometimes re-interpret them with new meanings. Goldschmidt (1991) observed this phenomenon, "seeing-as" activity in her terminology, in the study on architectural design processes. Goel (1995) called this "lateral transformation" in the study on graphic design processes. The significance of these studies lies in the revelation of important aspects of design sketches. They have not clarified, however, the functions of design sketches from the following viewpoints. What is the structure of designers' actions during the process? How is the structure formed at each local design stage? How are sketches involved in the formation of the structure?

Our aim was to address these issues with the expectation that the results would provide insights into what sort of computational tools should support design processes and how. Most available tools support design processes when designers need to efficiently visualize, compare and evaluate design ideas that they have already developed. Few tools are available to support the very process by which designers make sketches and thereby give birth to promising design ideas. Electronic pen-based sketching tools have received increasing interest recently (e.g. Kramer, 1994; Gross, 1995). Most tools are designed, however, on the basis of conjectured models of design processes or on findings about particular aspects of the role of sketches. They are not based on empirical modelling grounded in the ways designers draw, see and think.

With this goal in mind, we have analyzed the cognitive processes of designers. Protocol analysis has been the major technique to examine cognitive processes in design (e.g. Akin and Lin, 1995; Eastman, 1970; Eckersley, 1988; Gero and McNeill, 1998; Goldschmidt, 1991; McGinnis and Ullman, 1992; Schon and Wiggins, 1992; Suwa and Tversky, 1996, 1997b). We have devised a new scheme that allows us to code cognitive actions of designers from their video/audio protocols. After reviewing previous protocol analysis methods, the basic concept of our coding scheme will be described in Section 3. Based on the coding scheme, we analyzed the design process of a practicing architect. The results from the analysis have given us insights into how sketches facilitate design processes. Further they have significant implications for the types of computer tools needed to support sketching processes. 


\section{Previous Protocol Analysis Methods}

According to Dorst and Dijkhuis (1995) protocol analysis methods are divided into two categories; the process-oriented approach and the content-oriented approach. The former approach focuses on describing design processes in terms of a general taxonomy of problem-solving, i.e. problem-states, operators, plans, goals, strategies, and so on (e.g. Akin, 1993; Chan, 1990; Eastman, 1970; Kraus and Myer, 1970; Purcell et. al, 1994). The content-oriented approach aims at revealing the contents of what designers see, attend to, think of and retrieve from memory while designing (e.g. Goldschmidt, 1991; Schon and Wiggins, 1992; Suwa and Tversky, 1996, 1997b). Since we are interested in understanding the role of sketches in design, we have taken the content-oriented approach.

Suwa and Tversky $(1996$; 1997b) classified the contents of what designers see, attend to, and think of into four information categories; depicted elements and their perceptual features, spatial relations, functional thoughts, and knowledge. The first two are visual information, while the latter two are inherently non-visual. An important feature of this classification is that there are inherent dependencies between pieces of information belonging to different categories. For example, functional thoughts are suggested by attention to visual information in the sketch. Attention to a spatial relation between two regions in a sketch is based on inspecting the physical depiction of each region. Dependencies of this sort are the key to understanding how designers perceive visuo-spatial information from their sketches and how they use it for exploring non-visual functional issues which design thoughts center around. Suwa and Tversky's categories, however, were not necessarily developed for precisely capturing all the potential dependencies of this type. For example, the category called "depicted elements and their perceptual features" includes not only evidence that designers depicted elements but also one that they perceived the shapes or sizes of depicted elements. From the viewpoint of the dependencies mentioned above, however, depicting and perceiving should be distinguished from each other. We have devised a new set of categories, still inheriting much of their concept.

\section{The Coding Scheme}

This coding scheme has been developed from intensive observation of video/audio protocols of a practicing architect. We used data that Suwa and Tversky collected in their experiment (1997a). The task given to participants in the experiment was to design an art museum on a given site. They worked on the task for 45 minutes while sketching on sheets of tracing paper. Their sketching activities were videotaped. After the design task, they watched the 
videotape and reported what they remembered they had been thinking of when drawing each stroke of their sketches.

\subsection{ANALYSIS OF THE PROTOCOLS}

\subsubsection{Segmentation}

Using the standard protocol analysis methods, we segmented the entire verbal protocols into small units. We carried out segmentation in such a way that a change in the participant's intention, the content of his or her thoughts or actions flags the start of a new segment (Gero \& McNeill, 1998; Goldschmidt, 1991; Suwa \& Tversky 1997b; Van Someren et. al, 1994). A single segment sometimes consists of one sentence and sometimes of many.

\subsubsection{Action Categories}

For each segment, we code the cognitive actions of designers into four categories; physical, perceptual, functional and conceptual. This classification is similar to but differs slightly from Suwa and Tversky's coding scheme. We devised the present categories so that they correspond more precisely to the levels at which incoming information is processed in human cognition; information coming into human cognition is processed first sensorily, then perceptually and then semantically. Physical actions correspond to the sensory level, perceptual actions to the perceptual, and both functional and conceptual actions to the semantic level.

The first category, physical, refers to actions that are directly relevant to physical depictions on paper. There are three types of actions. One is to make depictions on paper, such as diagrams, symbols, annotations, memos, and sentences. We call these 'D-actions' (see Table 1 for a summary of all actions). The second is the motion of a pencil or hands that do not end up with depictions. We call these 'M-actions'. The last type is to look at existing depictions. We call these 'L-actions'.

The second category, perceptual, refers to reading off perceptual features perceivable from depictions. They are the shapes, sizes, textures of depicted elements and spatial relations among elements. We call these 'P-actions'. For example, if a designer draws a new depiction near an existing one by attending to the spatial relation between both depictions, then we code the new depiction as a D-action, his attention to the existing depiction as a L-action, and his attention to the spatial relation as a $\mathrm{P}$-action. This piece of $\mathrm{P}$-action is viewed as having occurred dependent on both $\mathrm{D}$ - and L-actions. This way, in principle, Pactions are inherently dependent on physical actions.

The third category, functional, refers to thinking of the functions or meanings with which designers associate physical depictions and/or their perceptual features. We call these 'F-actions'. For example, if a designer attends 
to a spatial relation between two regions and associates it with "views" from and to both places, we code his thought on "views" as a F-action. This F-action is viewed as having occurred dependent on the P-action to perceive the spatial relation. In principle, functions or meanings are not actually given in the appearance of perceptual features in sketches, but suggested by it. This way, Factions are dependent on physical and/or perceptual actions.

Table 1. Action categories

\begin{tabular}{lllll}
\hline categories & description & subtypes & name & examples \\
\hline \hline \multirow{2}{*}{ physical } & $\begin{array}{l}\text { relevant to } \\
\text { physical } \\
\text { depictions }\end{array}$ & $\begin{array}{l}\text { make depictions } \\
\text { look at depictions } \\
\text { other actions }\end{array}$ & $\begin{array}{l}\text { D-action } \\
\text { L-action } \\
\text { M-action }\end{array}$ & lines, symbols, words \\
\hline perceptual & read off perceptual & & P-action & shapes, sizes, textures, \\
& information & & & spatial relations \\
\hline \multirow{3}{*}{ functional } & associate visual & & F-action & $\begin{array}{l}\text { concepts such as "views", } \\
\text { information with }\end{array}$ \\
& meanings & & "flow of people", "light" \\
\hline \multirow{2}{*}{ conceptual } & higher & evaluate decisions & E-action & "I like this layout!" \\
& cognition & set up goals & G-action & \\
& & retrieve knowledge & K-action & \\
\hline
\end{tabular}

The fourth category, conceptual, refers to actions that are not dependent on physical and/or perceptual actions. There are three types of actions. The first one is to evaluate perceptual and functional information. We call these 'Eactions'. For example, if a designer evaluated as excellent a spatial pattern in which people flow within a site, we code his evaluation, "excellent", as an Eaction. The second type is to set up goals. We call these 'G-actions'. A goal is sometimes born in a bottom-up way, triggered by perceptual or functional actions. Or, a goal is sometimes set up in a top-down way, triggered by an existing goal, as the subgoal of the existing one. It occurs when a designer divides the current problem into subproblems to carry out the existing goal. Once a goal is set up, it in turn gives birth to other actions, i.e. G, F, or Pactions, in a top-down way. The third type is retrieval of knowledge from memory. We call these 'K-actions'. Knowledge is retrieved for carrying out reasoning.

\subsection{AN EXAMPLE OF THE CODING SCHEME}

Figure 1 is an excerpt of the protocols of a practicing architect 10 minutes into the design task. He had spent the very beginning period of his design process on 
estimating the required sizes of a museum building and a parking lot as 40,000 and 80,000 sq. ft. respectively. And just before coming to this excerpt, he had depicted a rectangle for the parking lot with that size. This excerpt describes his thoughts when he was about to draw a region for the museum building. He drew it, attending to the relationship at this moment that the building should be half the size of the parking lot.

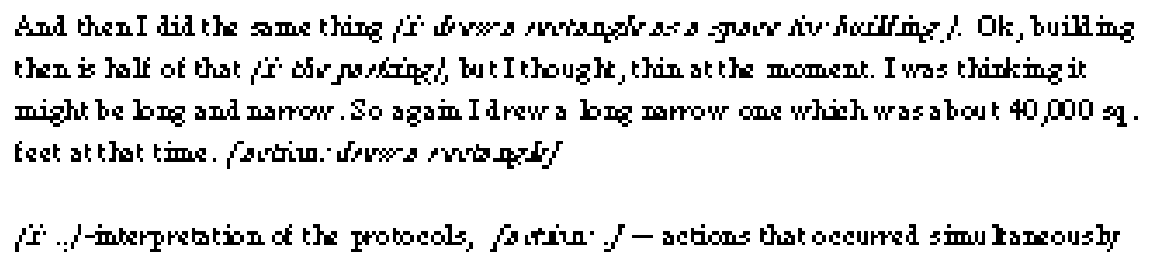

Figure 1. An excerpt of the protocols of a practicing architect.

We coded his thoughts in this excerpt in the following way. While looking at the memo, "40,000 sq. ft." ( $\mathrm{L}_{1}$ in Fig. 2), that he had left on a sketch, and interpreting it again as the size of the building $\left(\mathrm{F}_{1}\right)$, he set up a goal, "draw a building of this size on paper" $\left(\mathrm{G}_{1}\right)$. His words, "OK, building is then ..", suggest the existence of this goal. Because he noticed that the building should be half the size of the parking lot $\left(\mathrm{F}_{3}\right)$, he decided to cut the rectangle for the parking lot in half. We interpret that he divided the problem into two subproblems; looking at the rectangle and creating a new depiction with half the size. Both actions were initiated by the subgoals $\left(\mathrm{G}_{2}\right.$ and $\left.\mathrm{G}_{3}\right)$ that have been set up under the original goal. Attention to the relation between the sizes of both $\left(\mathrm{F}_{3}\right)$ triggered a piece of domain-independent strategy $\left(\mathrm{K}_{2}\right)$ for setting up those subgoals. $\mathrm{G}_{2}$ triggered the action to look at the rectangle for the parking lot. $\mathrm{G}_{3}$ triggered the action to draw the rectangle $\left(\mathrm{D}_{1}\right)$ with half the size $\left(\mathrm{P}_{1}\right)$. The shape happened to be thin and narrow $\left(\mathrm{P}_{2}\right)$. Of course, he associated the new rectangle with "building" function.

Figure 2 is a schematic diagram that shows the structure of his cognitive actions in this excerpt in terms of the relations among his cognitive actions. Each line in Figure 2 represents the inherent dependency between a pair of two actions and each arrow the triggering relation. 


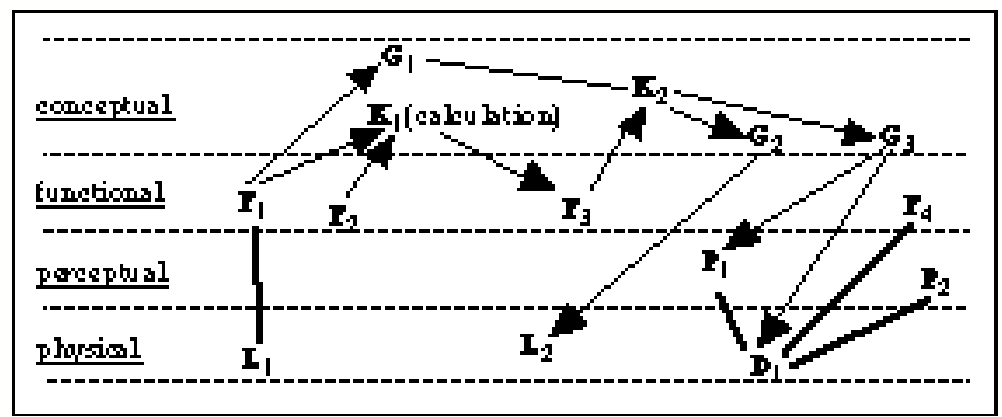

Figure 2. The schematic diagram representing the structure of design actions

\section{Analysis of Designer's Cognitive Behaviours}

Through interaction with sketches at the physical level, designers are then able to have higher interaction at the perceptual and functional levels. This way, information "emerges" in designers' perception in a bottom-up way. We conjectured that this bottom-up process is a key to understanding how sketches function in design processes. On the other hand, processes involving conceptual actions are different in nature. They are a top-down control over subsequent actions. Although top-down processes are also worth while examinations, it is beyond the scope of this paper. Our examination here is limited to the lower three levels of actions.

\subsection{DOMINANT COGNITIVE ACTIONS}

We examined the frequency of the occurrence of functional, perceptual and physical actions throughout the process of the architect. He produced seven sheets of sketches, Figure 3. He stated that each page represented a distinct design phase. Pages 1 and 2 involved analysis of the site and the design requirements. Page 3 was the phase to roughly arrange functions within the site. All the subsequent pages were based on this arrangement. In Page 4, called "scheme A", the architect explored design decisions in more detail. In Page 5, called "scheme B", he explored in another way. In Page 6, called "scheme B plan", he focused on the building plan based on Page 5. In Page 7, called "scheme A plan", he focused on the building plan based on Page 4. 
$\mathrm{P} \approx \mathrm{g} \times 7$

Figure 3. The seven pages of design sketches of a practicing architect. 
For each page, we calculated the sum total of occurrences of the three types of actions. Figure 4 shows, for each page, the ratio of each type of actions to the total. In Pages 1 and 2, physical actions were dominant while functional actions were less frequent. In Page 3, functional actions occurred more frequently than in Pages 1 and 2, and consequently physical actions were less dominant. In Pages 4, 5 and 6, this tendency was more salient. In Page 7, functional actions became less frequent again, while physical actions increased a little. The three circular shapes in Page 7 were intended for a set of museum buildings. In the first half of Page 7, the architect worked on a new spatial arrangement of these three, discarding some of the basic arrangement he had made in Page 3. So, Page 7 may have been closer to Page 3, the phase of spatial arrangement. This explains the tendency in Figure 4.

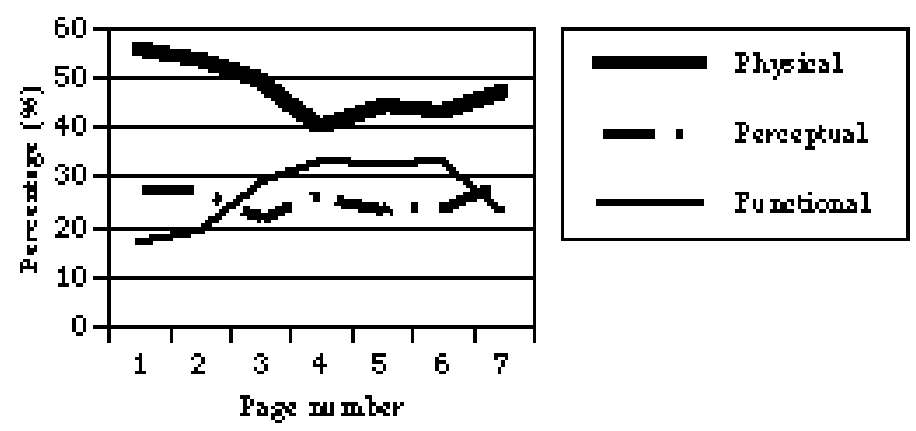

Figure 4. The percentage of each level of actions to the total, for each page of sketches.

The findings of this analysis are as follows. First, the design process contains three distinct phases, problem analysis, spatial arrangement, and functional exploration. Second, the occurrences of functional actions and physical actions capture the characteristic of each design phase. Functional actions dominate in the phase of detailed functional exploration. Physical actions are dominant in the phase of problem analysis. The phase of spatial arrangement is intermediate between the two.

\subsection{CORRELATION OF DIFFERENT TYPES OF ACTIONS}

\subsubsection{Correlation between P-actions and others}

We investigated how the occurrences of different types of actions correlate with each other throughout the design process. If the occurrences of two particular types of actions correlate with each other in certain periods, it suggests that the inherent dependency between the two was exclusive and salient in those periods. This examination is expected to reveal how the designer cognitively 
interacted with his own sketches. Figure 5 shows how the frequencies of occurrences of P-actions and L-actions changed over time throughout the entire process. The horizontal axis is the segment number, representing the time frame in which the design process occurred. Shown on the bottom are the periods of time corresponding to the pages of the designer's sketches. The vertical axis represents the frequency of the occurrences of each type of action. We normalized the actual frequency by its average as follows; the vertical value is $\left(F_{X}-F_{X} a v g\right) / F_{X} a v g$, where $F_{X}$ is the actual frequency of $X$-actions and $F_{X} a v g$ is the average frequency throughout the process. The normalization is intended to compare both actions after removing the magnitude of frequency specific to each type. Since segment-by-segment changes of the frequency of actions might be too sharp to be tractable for comparison, we chunked every five segments from the beginning of the protocols, and calculated the sum total of the occurrences of each type of actions for each 5-segment period.

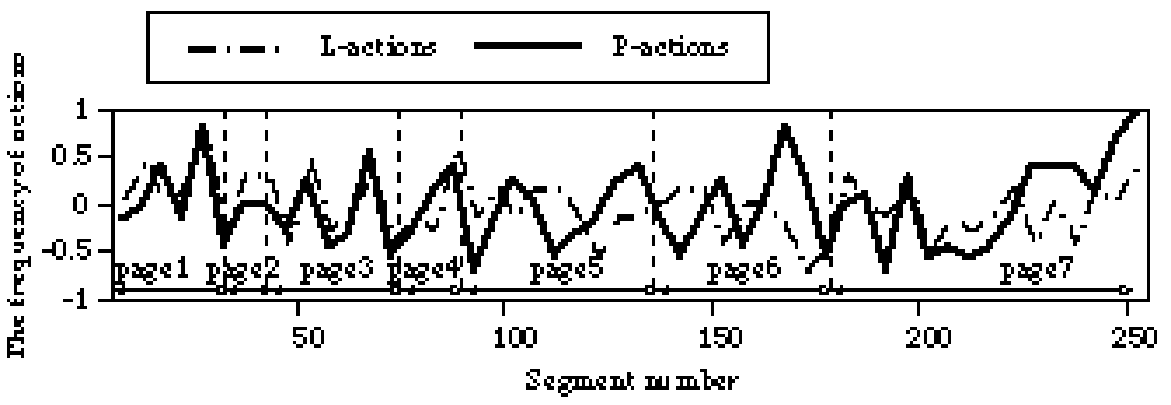

Figure 5. The correlation between P-actions and L-actions.

If P-actions vary in correlation with L-actions in certain periods, it suggests the following. First, looking at existing depictions most induced perceptual actions to occur simultaneously in these periods. Second, the majority of perceptual actions occurred by being triggered by looking at existing depictions, although perceptual actions could be potentially dependent on any physical actions, i.e. L-, D-, or M-actions. In Figure 5, P-actions and L-actions correlate from the second half of Page 1 through the end of Page 3, and in the first half of Page 7.

We examined the correlation of two different actions, for the following pairs of actions as well; P- vs. D-actions, P- vs. M-actions, D- vs. L-actions, F- vs. Pactions, F- vs. L-actions, F- vs. D-actions, F- vs. M-actions. For each pair, we performed statistical analyses to identify the periods in which there is a correlation between the two. For each 5-segment period, we calculate the difference of the frequency of actions from its immediately previous period. Then, we identify the periods in which the differences of a type of actions correlate with those of the other type for more than or equal to two consecutive 
transitions from a 5-segment period to the subsequent period. This way, such periods in which both types of actions happen to increase or decrease in the same direction only for a single transition are eliminated. We do this by conducting $\chi$-square tests on the pair of the consecutive period-to-period differences. Only periods in which the correlations are statistically valid with a certainty of more than $90 \%$ are considered.

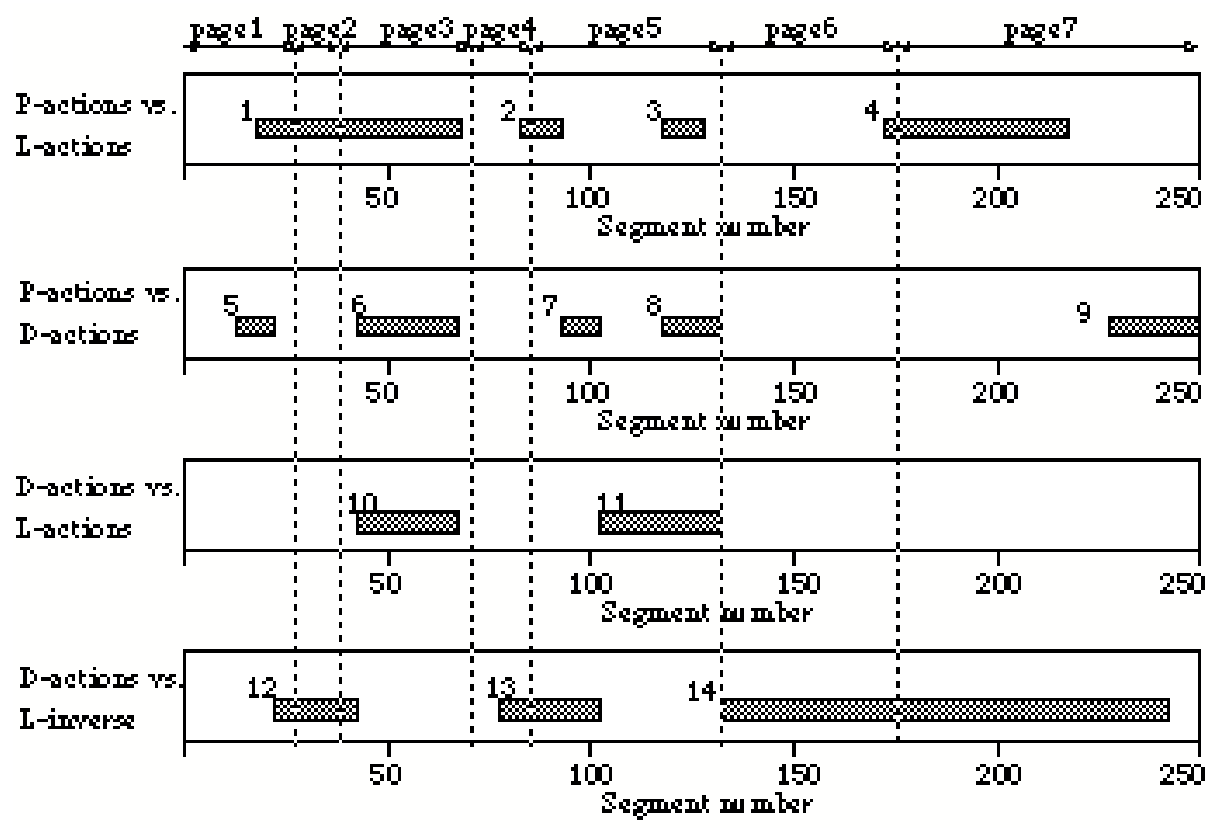

Figure 6. The portions of design process in which there are correlations between two actions for the pairs that are concerned with $P$-actions.

Figure 6 shows, for each pair of actions that are concerned with P-actions, the periods in which there was a correlation. The horizontal axis is the segment number, thus representing the time frame. The horizontal bars show the periods of correlation. The number written beside each bar is the identification number of the period, corresponding to each ID number in Table 2. Table 2 shows, for each period of correlation, the corresponding statistical data, i.e. the duration of the period in terms of the number of consecutive transitions, a $\chi$-square value, and a certainty. The pairs of actions for which there was no correlation throughout the process are not shown here. For D- and L-actions, we examined whether or not both actions have a negative effect on each other, by performing the same statistical analysis on the pairs of the period-to-period differences of $\mathrm{D}$-actions and the inverse period-to-period differences of L-actions.

Table 2. The statistical data of portions of correlation 


\begin{tabular}{|c|c|c|c|c|c|c|c|}
\hline $\begin{array}{l}\text { ID. of } \\
\text { portion }\end{array}$ & $\begin{array}{c}\text { consecutive } \\
\text { transitions } \\
\end{array}$ & $\begin{array}{c}\chi \text {-square } \\
\text { value } \\
\end{array}$ & $\begin{array}{c}\text { certainty } \\
(\mathrm{p}>)\end{array}$ & $\begin{array}{c}\text { ID. of } \\
\text { portion }\end{array}$ & $\begin{array}{c}\text { consecutive } \\
\text { transitions }\end{array}$ & $\begin{array}{c}\chi \text {-square } \\
\text { value }\end{array}$ & $\begin{array}{c}\text { certainty } \\
(\mathrm{p}>)\end{array}$ \\
\hline 1 & 10 & 0.699 & 0.995 & 15 & 3 & 0.457 & 0.9 \\
\hline 2 & 2 & 0.160 & 0.9 & 16 & 7 & 0.972 & 0.995 \\
\hline 3 & 2 & 0.140 & 0.9 & 17 & 2 & 0.171 & 0.9 \\
\hline 4 & 9 & 2.08 & 0.99 & 18 & 2 & 0.162 & 0.9 \\
\hline 5 & 2 & 0.039 & 0.975 & 19 & 4 & 0.549 & 0.95 \\
\hline 6 & 5 & 0.526 & 0.99 & 20 & 5 & 0.704 & 0.975 \\
\hline 7 & 2 & 0.012 & 0.99 & 21 & 3 & 0.456 & 0.9 \\
\hline 8 & 3 & 0.088 & 0.99 & 22 & 3 & 0.310 & 0.95 \\
\hline 9 & 4 & 0.880 & 0.9 & 23 & 4 & 0.291 & 0.99 \\
\hline 10 & 5 & 1.19 & 0.95 & 24 & 2 & 0.136 & 0.9 \\
\hline 11 & 6 & 1.84 & 0.9 & 25 & 2 & 0.062 & 0.95 \\
\hline 12 & 4 & 0.680 & 0.95 & 26 & 5 & 0.900 & 0.95 \\
\hline 13 & 5 & 0.950 & 0.95 & 27 & 3 & 0.404 & 0.9 \\
\hline \multirow[t]{2}{*}{14} & 22 & 8.80 & 0.99 & 28 & 2 & 0.198 & 0.9 \\
\hline & & & & 29 & 5 & 0.631 & 0.975 \\
\hline
\end{tabular}

There are two periods in which P-actions correlated with both L- and Dactions; almost the entire part of Page 3 and the end of Page 5. In these periods, the designer drew and looked at existing depictions simultaneously, and both induced his perceptual actions. The majority of perceptual actions which occurred were dependent on both actions. This may be characteristic of the phase of spatial arrangement in which things are arranged on a sketch by attending to the spatial relations between themselves and existing depictions. We recognized in his protocols that he spent the ending portion of Page 5 on arranging sculptures and ponds in the remaining area of his sketch, after he had explored the details of the building plan.

The periods in which P-actions correlated with L-actions only and not with $\mathrm{D}$-actions were longer that those in which P-actions correlated with D-actions only. The former periods cover $32 \%$ of the entire process, while the latter $16 \%$. This suggests that perceptual actions were more likely to occur later when he inspected existing depictions in a "revisited" way than simultaneously when he was making depictions, except for the phase of spatial arrangement.

For almost all the parts of the process except for the phase of spatial arrangement, drawing and looking at existing depictions have a negative effect on each other. This clearly indicates a separation between drawing and inspecting. If drawing becomes frequent, looking at existing depictions becomes less frequent, and vice versa.

These findings about P-actions have led to the following insight. Except for the phase of spatial arrangement, the role of drawing is to leave ideas down on a 
sketch as visual tokens, so that they can be revisited later for inspection. This inspection will then stimulate perception.

\subsubsection{Correlation between F-actions and others}

Figure 7 shows that F-actions correlated with P-actions from the last half of Page 2 through the beginning of Page 3, for almost the entire part of Pages 4 and 5, and for the latter half of Page 7. During these periods, the major way in which F-actions occurred was to associate visuo-spatial features with functional issues, although F-actions could potentially occur by being suggested by physical actions without mediation of P-actions. This means that visuo-spatial information perceivable from sketches became the cues for association of functional information during these periods.

The important characteristic true to all the three major occurrences of correlations between F- and P-actions is that it came after a correlation between $\mathrm{P}$ - and L-actions had lasted for a while. This finding has an implication for the conditions and ways in which visuo-spatial information becomes the cues for association. This will be discussed in more detail in the next section.

Past literature suggested that unexpected discoveries are one of the benefits from using sketches. We successfully identified them as a particular type of Pactions based on our coding scheme. We examined how F-actions correlated with unexpected discoveries. We did not conduct statistical analysis for this, because the frequency of the occurrence of unexpected discoveries is relatively fewer than F-actions, and the normalization mentioned earlier tends to exaggerate the fluctuation of unexpected discoveries more than F-actions. Instead, we visually analyzed the correlation between both. Figure 8 shows, for Pages 4 and 5, the frequency of F-actions, unexpected discoveries, and the remaining P-actions. The vertical axis is the actual frequency. The peaks of Factions correlated more with the increase of unexpected discoveries than with that of the remaining types of P-actions. In the latter half of Page 7, where Factions correlate with P-actions, there was no such tendency. 


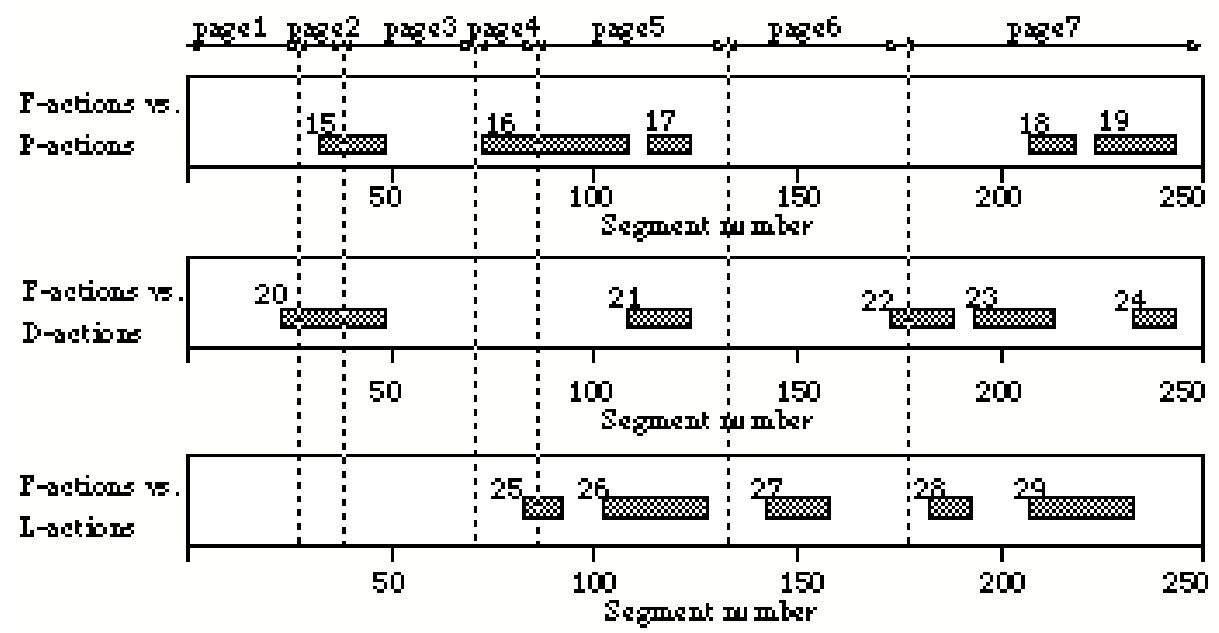

Figure 7. The portions of design process in which there are correlations between two actions for the pairs that are concerned with F-actions.

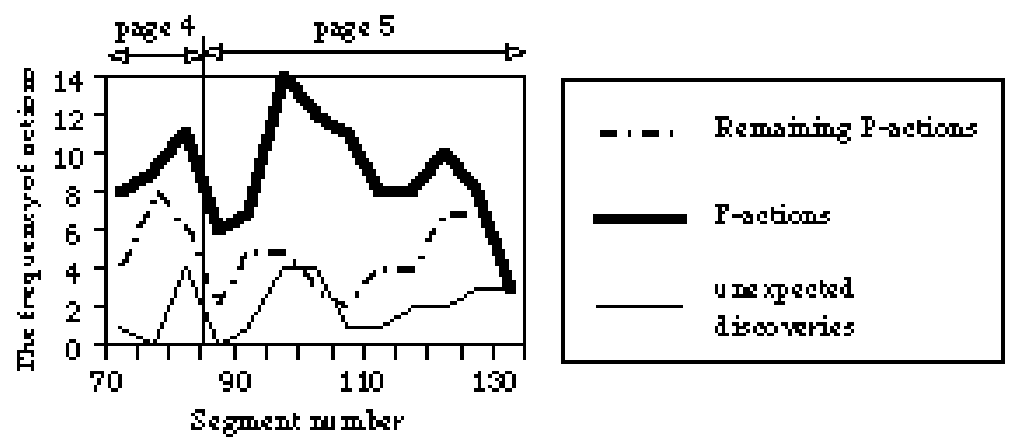

Figure 8 . The correlation between $F$-actions, unexpected discoveries and the remaining $P$ actions.

F-actions correlated with D-actions only without the mediation of P-actions, in the beginning of Page 2 and in the first half of Page 7. The majority of functional thoughts occurred in such a way that the designer named what he was drawing. F-actions correlated with L-actions only, for some portions of Page 6. The majority of functional thoughts occurred in such a way that he remembered the meanings of depictions when he re-looked at them. The duration of these correlations, however, were much shorter than the periods of correlation between F- and P-actions.

These findings about F-actions have led to the following insights. First, the phase of functional exploration can be characterized by the phenomenon that 
the majority of functional thoughts occur by being suggested by perceptual actions, not just by physical actions. Designers perceive visuo-spatial features from sketches and use them as cues for thinking about non-visual functional issues. Second, perceptual features that are discovered in an unexpected way may sometimes, though not always, become the cues for functional issues. This is a hypothesis to be examined by seeking data on more designers.

\section{Discussion}

\subsection{DESIGN BEHAVIORS ARE SITUATED IN DESIGN SKETCHES}

The professionalism of expert designers has been attributed to their possession of domain knowledge and problem-solving strategies and to their ability to develop appropriate plans and goals. Thus, much emphasis has been put on the examination of how they use problem-solving strategies, plans, goals and knowledge. Most process-oriented protocol analysis addressed this issue. This tendency has been prevalent in the artificial intelligence community as well. Experts' intelligent behaviors are attributed to domain knowledge.

The present study, however, has shed light on another important factor in design. Knowledge, strategies, goals and plans do not always initiate or control design actions. Rather, perceptual and physical actions play central roles in many ways. Looking at existing depictions often induces perceptual actions, which in turn provide the cues for association of functional issues. In addition, association of functional issues does not always or randomly occur, but occurs only after absorption of perceptual features has lasted for a while. Put differently, perceptual actions serve as a preparation for functional actions. Physical and/or perceptual actions sometimes even trigger the set-up of goals or retrieval of knowledge, as we saw in Figure 2. These findings suggest that designers think not just in goal-directed or knowledge-intensive ways, but quite often construct their thoughts on the fly in response to depicted elements in sketches and their perceptual features. This coincides with the recently prevailing view that people's actions are situated in the physical setting they are in (Agre and Chapman, 1987; Kirsh, 1995). Designers' behaviours, too, are situated in the physical setting in which they make sketches and inspect them.

\subsection{A DESIGN PROCESS HAS DISTINCT PHASES}

Dominant actions change over the entire process. The ways in which different types of actions are correlated with each other are not uniform throughout the entire process. This clearly indicates that the entire design process comprises of distinct phases. We recognized at least three major phases: problem analysis, 
spatial arrangement, and detailed functional exploration. The phase of functional exploration could also have a distinct sub-phase in which designers check the design ideas that have been already explored.

\subsection{A DESIGN PROCESS IS NOT JUST ONE CYCLE}

Generally speaking, design phases tend to occur in a process in the following order. Problem analysis comes first. Detailed functional exploration comes after spatial arrangement has been gone through. It is not the case, however, that a design process consists of only one cycle of these phases, but that it consists of a series of cycles. The results shown in Figures 6 and 7 support this claim. Page 3 is clearly the phase of spatial arrangement, and the phase of detailed functional exploration came after that, in Pages 4 and 5. But, at the end of Page 5 , spatial arrangement came again, although for a short period of time. Page 7 consists of more than one phase. The first half of Page 7 is in a way close to the phase of problem analysis; there are correlations between P- and L-actions and between F- and D-actions. This means that he was drawing depictions by naming them and attending to perceptual features by looking at existing depictions. The second half of Page 7 is the phase of detailed functional exploration.

There are two reasons for the cyclic appearance of distinct phases. First, when an alternative idea is proposed and tested, it forms a new cycle of phases. Second, one page of sketches can contain many spatial regions and each of them could potentially represent a specific design phase different from the phases in other regions. The externality of sketches encourages designers to shift their attention from one part of a sketch to another when they are stuck in or done with exploration in one part, and consequently to shift from one phase to another.

Purcell et al's analysis (1994) is relevant to this view. They conducted a microscopic analysis on the strategies that designers employ in each segment. Their claim was that a designer's actions in analyzing problems, and synthesizing and evaluating solutions are not linearly ordered in a process, but rather repeated in a rapid series of cycles. Compared with this, our analysis is more macroscopic; we focused on design phases rather than the strategies used in each local segment. Design phases are not just linearly ordered but repeated in cycles.

\section{Implications for Ways to Support Design Processes}

The present study clearly indicates that design behaviours are situated in physical settings in which designers make drawings, look at existing drawings and read off perceptual information from them. This implies that success in the 
support of design processes is highly dependent on whether or not physical and perceptual actions of a designer are supported in appropriate ways.

First, support tools should have a natural interface that enables easy and quick drawing without cognitive burden. This requirement is magnified by our finding that drawing and looking at existing depictions have negative effects on each other, and perception tends to occur in correlation more with looking than with drawing. Cognitive burden in drawing would suppress the time for looking at existing drawings and thus hamper perception. Pen-based electronic sketching tools (e.g. Gross, 1996; Kramer, 1994; Landay and Myers, 1995) are promising in this respect.

Second, support tools should provide designers with the ability to manipulate depicted elements efficiently. In Kirsh's Tetris game example (1995), people tend to move horizontally and rotate dropping items for better perception. In the domain of architectural design, this corresponds to moving and rotating depicted elements in a sketch, and to revising the perceptual features of depicted elements, such as sizes, shapes and textures. This type of support may be useful to enhance perceptual actions in the phase of spatial arrangement, in which drawing mostly induces perceptual actions. In order not to hamper perception, depictions should be manipulable on a visual basis, without having to pay attention to the internal representation of elements in the tool. Whether or not old features before the manipulation should be left so that the tool will have multiple representations, and how it should be done require further study. These two are the support for drawing.

Third, support for looking at existing depictions is needed as well. Considering that perception occurs more often when designers look at existing depictions than when they draw, support tools should have a mechanism to encourage designers to revisit their own depictions as often as possible. For example, if an electronic sketching tool monitors what elements are drawn on the sketch pad and when, it may be able to present information about what depicted elements have not been revisited since they were drawn, or about which regions on the sketch the designer's pencil has revisited less frequently. The ability to superimpose visual stimuli on depicted elements themselves, e.g. by highlighting them using colors, would help arouse the designer's attention to the elements. Or, it may suffice to prepare buttons on the interface of the tool that are always ready to be hit by designers when they need to check nonrevisited elements or regions. Further study is needed, however, to address what interface is appropriate and, if a visual stimulus is appropriate, when it should be presented and how.

Fourth, support tools should have mechanisms to enhance perceptual interaction with physical depictions (e.g. Suwa and Gross, in press). If perceptual actions are enhanced, it may stimulate the occurrence of functional thoughts more often, as the present study suggests. For example, when a 
designer moves or revises the features of a depicted element, it might be useful to let him notice the spatial relation between the element and others that were depicted together with the element. A sketching tool that has the ability to monitor designer's sketches would be able to present visual stimuli to let him notice it. For another example, it might be fruitful to encourage designers to make unexpected discoveries on their own sketches. Arousing their attention to depicted elements that are arranged at adjacent locations in a sketch but were drawn in at different times in the process may be a useful means to encourage the discoveries of spatial relations in an unintended way. Or, reversing the figure/ground relationship in the image of a sketch so that the ground part of the original sketch will emerge may be useful to stimulate the discoveries of spaces as ground. Enhancement of perception of this sort, then, again highlights the importance of the first requirement, quick and easy drawing. When designers make unexpected discoveries, they may want to quickly represent what they have perceived on the sketch.

What is particularly important is that support tools should maintain the situatedness of the processes. We have proposed the idea of different classes of support here. However, the process in which designers' actions belonging to different cognitive levels intermingle with each other is vulnerable and its cycles are rapid. If any one of those methods badly affects the process and/or breaks its rapidity, or is contrary to the designers' approach, it will easily destroy the situatedness. Rather, support tools should be able to give designers incentives to draw and perceive while the situatedness of the entire cycles of their cognitive actions is maintained.

\section{Conclusion}

The goal in this paper was to analyze the cognitive processes of a designer in terms of how design sketches play a central role in the process, in order to provide a foundation for computational support. For this purpose, we devised a new scheme for coding designers' cognitive actions from the video/audio protocols. Their actions are coded into one of the four cognitive levels.

The analysis has brought us the following findings about the nature of design processes. First, the role of sketches is to leave ideas down on paper for later inspection, rather than inducing simultaneous perception. Perception occurs more while looking at existing depictions. Second, in the core of a design process in which designers explore alternative functional thoughts, the majority of functional thoughts are stimulated by perception rather than by physical actions directly. Third, a design process has distinct design phases each of which is characterized by the dominancy of particular types of cognitive actions and by dependency between them. Fourth, designers' behaviors are situated in 
the physical setting in which they draw, look at, and perceive. Sketches are thus essential to design processes.

These findings have implications for the types of support that computational tools should provide. One type of support is a quick and easy sketching tool that does not suppress the cognitive load necessary to look at depictions. The second type provides easy manipulation of depicted elements for better perception. The third type provides encouragement for looking at existing depictions. The fourth type provides opportunities for unexpected discoveries for stimulation of functional thoughts. Different types of support are needed in different design phases. The most important principle is to always maintain the situatedness of design behaviors. These tools to support designers' cognitive processes define areas of further artificial intelligence development in design.

\section{Acknowledgments}

We are grateful to B. Tversky of the Department of Psychology, Stanford University for insightful discussions about human cognition. The discussion with M. D. Gross of University of Colorado, Boulder has been stimulating to our current ideas about computational support tools. This research has been funded by an Australian Research Council Grant, Number A89601894.

\section{References}

Akin, O.: 1993, Architects' reasoning with structures and functions, Environment and Planning B: Planning and Design, 20, 273-294.

Akin, O. and Lin, C.: 1995, Design protocol data and novel design decisions, Design Studies, 16(2), 211-236.

Agre, P.E., and Chapman, D.: 1987, Pengi: an implementation of a theory of activity, Proceedings of AAAI-87, Seattle, WA, pp.268-272.

Chan, C-S.: 1990, Cognitive processes in architectural design problem solving, Design Studies, 11(2), 60-80.

Dorst, K and Dijkhuis, J.: 1995, Comparing paradigms for describing design activity, Design Studies, 16(2), 261-274.

Eastman, C. M.: 1970, On the analysis of intuitive design processes, in G. T. Moore (ed.), Emerging methods in environmental design and planning, MIT Press, Cambridge, pp.21-37.

Eckersley, M.: 1988, The form of design processes: a protocol analysis study, Design Studies, 9(2), 86-94.

Gero, J. and McNeill T.: 1998, An approach to the analysis of design protocols, Design Studies, 19(1), 21-61.

Goel, V.: 1995, Sketches of thought. MIT Press, Cambridge.

Goldschmidt, G.: 1991, The dialectics of sketching, Creativity Research Journal, 4(2), 123-143.

Gross, M. D.: 1996, The electronic Cocktail Napkin - a computational environment for working with design diagrams, Design Studies, 17(1), 53-69.

Kirsh, D.: 1995, The intelligence use of space, Artificial Intelligence. 73(1,2), 31-68. 
Kramer, A.: 1994, Translucent patches - dissolving windows-, Proceedings of UIST'94. ACM, New York, pp.121-130.

Krauss, R. I. and Myer, R. M.: 1970, Design: a case history, in G. T. Moore (ed.) Emerging methods in environmental design and planning, MIT Press, Cambridge, pp.11-20.

Landay, J. A. and Myers, B. A.: 1995, Interactive sketching for the early stages of user interface design, Human factors in computing systems: proceedings of CHI'95, ACM, New York, pp. 43-50.

McGinnis, B. D. and Ullman, D. G.: 1992, The evolution of commitments in the design of a component, Journal of Mechanical Design, 114(1), 1-7.

Purcell, T., Gero, J., Edwards, H., and McNeill, T.: 1994, The data in design protocols: the issue of data coding, data analysis in the development of models of the design process, in J. S. Gero and F. Sudweeks (eds.), Artificial Intelligence in Design '94, Kluwer, pp. 225-252.

Schon, D. A. and Wiggins, G.: 1992, Kinds of seeing and their functions in designing, Design Studies, 13(2), 135-156.

Stenning, K. and Oberlander, J.: 1995, A cognitive theory of graphical and linguistic reasoning: logic and implementation, Cognitive Science, 19(1), 97-140.

Suwa, M. and Gross, M. D.: in press, Enriching perception: a new paradigm for design sketching tools, submitted to Asia Pacific Human Computer Interaction'98.

Suwa, M. and Tversky, B.: 1996, What architects see in their design sketches: implications for design tools, Human factors in computing systems: CHI'96 conference companion, ACM, New York, pp.191-192

Suwa, M. and Tversky, B.: 1997a, How do architects interact with their design sketches in exploring design ideas? Proceedings of 4th Australasian Cognitive Science Conference '97, Newcastle, Australia, to appear.

Suwa, M. and Tversky, B.: 1997b, What do architects and students perceive in their design sketches?: A protocol analysis, Design Studies, 18(4), 385-403.

van Someren, M. W., Barnard, Y. F. and Sandberth, J. A. C.: 1994, The think aloud method: A practical guide to modelling cognitive processes, Academic Press, London.

This is a copy of the paper: Suwa, M., Gero, J. S. and Purcell, T. (1998). Analysis of cognitive processes of a designer as the foundation for support tools, in J. S. Gero and F. Sudweeks (eds), Artificial Intelligence in Design' '98, Kluwer, Dordrecht, pp. 229-248. 